\title{
IMP3 and p16 expression in squamous cell carcinoma of the head and neck: A comparative immunohistochemical analysis
}

\author{
MARC-OLIVER RIENER ${ }^{1,2}$, JOSEF HOEGEL ${ }^{3}$, HEINRICH IRO $^{4}$, ARNDT HARTMANN $^{1}$ and ABBAS AGAIMY ${ }^{1}$ \\ ${ }^{1}$ Institute of Pathology, University Hospital, Friedrich-Alexander University Erlangen-Nuremberg, \\ D-91054 Erlangen; ${ }^{2}$ OptiPath, Pathology Joint Practice, Frankfurt D-60487; ${ }^{3}$ Institute of Human Genetics, \\ University Hospital Ulm, D-89081 Ulm; ${ }^{4}$ Department of Otorhinolaryngology, Head \& Neck Surgery, \\ Friedrich-Alexander University Erlangen-Nuremberg, University Hospital, D-91054 Erlangen, Germany
}

Received May 23, 2016; Accepted November 17, 2016

DOI: $10.3892 / \mathrm{ol} .2017 .6352$

\begin{abstract}
Expression of p16 has been established as a good surrogate marker for high-risk human papillomavirus (HPV) infection in oropharyngeal squamous cell carcinoma (OPSCC) patients, and it has been associated with an improved prognosis, irrespective of the actual HPV status. Conversely, the human insulin-like growth factor II mRNA binding protein 3 (IMP3) has been related to aggressiveness in several types of tumors. The aim of the present study was to investigate and compare p16 and IMP3 as markers of favorable and unfavorable behavior, respectively, in head and neck SCC (HNSCC), with particular reference to the HPV status. Both markers were analyzed by immunohistochemical analysis of 156 HNSCC samples originating from the oropharynx $(n=81)$, oral cavity $(n=44)$, larynx $(n=15)$, hypopharynx $(n=10)$ and nasopharynx $(n=6)$. The HPV status was examined in a randomly selected representative subcohort $(\mathrm{n}=38)$ using polymerase chain reaction. Of the 156 HNSCC samples, 81 (51.9\%) and 54 (34.6\%) were positive for IMP3 and p16, respectively. IMP3 expression $(\mathrm{P}=0.022)$, p16 expression $(\mathrm{P}<0.001)$ and the combination of these markers $(\mathrm{P}<0.001)$ were significantly associated with tumor site. In particular, 69/81 (85\%) OPSCC samples were positive for either one or both markers compared with 36/75 (48\%) SCC samples from other sites. p16 expression was significantly associated with HPV infection $(\mathrm{P}=0.017)$ and a trend towards a negative association between IMP3 expression and HPV infection was observed $(\mathrm{P}=0.053)$. The results of the present study suggested that IMP3 and p16 are more frequently expressed in OPSCC compared with
\end{abstract}

Correspondence to: Professor Abbas Agaimy, Institute of Pathology, University Hospital, Friedrich-Alexander University Erlangen-Nuremberg, Krankenhausstraße 8-10, D-91054 Erlangen, Germany

E-mail: abbas.agaimy@uk-erlangen.de

Key words: insulin-like growth factor II mRNA binding protein 3, p16, immunohistochemistry, human papillomavirus, head and neck squamous cell carcinoma other HNSCCs. The prognostic impact of IMP3 on OPSCC remains to be investigated in a larger series with an extended follow-up period.

\section{Introduction}

Squamous cell carcinoma (SCC) is the leading cancer type affecting the head and neck region (HNSCC) representing $>90 \%$ of malignant neoplasms of the oral cavity and oropharynx and 5 and $2 \%$ of all cancers in men and women, respectively (1). Tobacco smoking and alcohol consumption are the two major etiological factors of HNSCC and they work in a synergistic manner to initiate SCC (1). Approximately $75 \%$ of HNSCC cases in western countries are related to these two major risk factors, while the remainder is predominantly associated with viral infection, in particular infection with high-risk human papillomavirus (HPV) (1). HPV has been detected in a small fraction of oral cavity SCCs, and in 40-50\% of oropharyngeal (mainly tonsillar) SCC (OPSCC) (1). While HPV-associated HNSCC may occur at any site, including the sinonasal tract, the majority of HPV-related SCCs originate in the lymphoid tissue bearing the oropharynx, mainly the palatine tonsil and the base of the tongue (1). Expression of p16 as a consequence of oncogenic HPV infection has been increasingly used as a surrogate marker for high-risk HPV infection, particularly in the female genital tract and the oropharynx $(2,3)$. The sensitivity and specificity of p16 immunostaining for detecting high-risk HPV in OPSCC patients has been in the range of 95-98\% (2,4-6). However, the value of p16 immunohistochemistry as a surrogate marker for HPV infection in head and neck sites other than the oropharynx, including the oral cavity, larynx, hypopharynx and sinonasal tract, has yet to be determined.

The human insulin-like growth factor II mRNA binding protein 3 (IMP3), which was originally named $\mathrm{KH}$ domain-containing protein overexpressed in cancer, plays an important role in early human embryogenesis, although it is commonly expressed only at low levels in adult tissues. IMP3 is considered an oncofetal protein that is expressed in fetal and carcinogenic tissue; however, the exact function of IMP3 remains unknown (7-12). Expression of IMP3 
has been studied in a wide variety of cancers, including renal cell carcinoma $(13,14)$, adenocarcinoma of the uterine cervix (15), endometrial carcinoma (16), adenocarcinoma of the esophagus (17), malignant melanoma (18), Merkel cell carcinoma (19), urothelial carcinoma (20), neuroendocrine carcinoma of the lung (21), gastric adenocarcinoma (22), hepatocellular carcinoma (23), pancreatic (24-27) and biliary tract (27) adenocarcinoma, and triple negative breast cancer (28), where this marker has frequently been associated with enhanced tumor aggressiveness and a worse outcome.

However, only a few studies have previously investigated the expression of IMP3 in head and neck SCC from specific sites (29-34). In particular, the pattern of IMP3 expression in HNSCC with regards to the HPV status has not previously been investigated. The aim of the current study was to compare the expression pattern of IMP3 (as a 'bad' marker) with that of p16 (as a 'good' marker) in HNSCC, with special reference to a patient's HPV status.

\section{Materials and methods}

Patients and tissue samples. The present study involved 156 consecutive patients diagnosed with HNSCC at the Institute of Pathology, University Hospital, Friedrich-Alexander University Erlangen-Nuremberg (Erlangen, Germany) and the OptiPath, Pathology Joint Practice (Frankfurt, Germany) between January 1998 and December 2015. The localizations of the SCCs were as follows: $81(51.9 \%)$ from the oropharynx, $44(28.2 \%)$ from the oral cavity, $15(9.6 \%)$ from the larynx, $10(6.4 \%)$ from the hypopharynx and $6(3.9 \%)$ from the nasopharynx. Only biopsy specimens were used in this study. The present study was performed in accordance with accepted principles of ethical and professional conduct for biomedical scientific research, and it was approved by the ethical committee of the medical faculty of the Friedrich-Alexander University Erlangen-Nuremberg for retrospective translational research activities.

Formalin-fixed (overnight at room temperature) and paraffin-embedded biopsy specimens were cut into sections (3- $\mu \mathrm{m})$ and mounted onto Superfrost ${ }^{\mathrm{TM}}$ slides (Menzel-Gläser; Thermo Fisher Scientific, Inc., Braunschweig, Germany). All biopsies were stained with hematoxylin and eosin on eight step sections. Immunohistochemistry with anti-IMP3 (clone 69,1; dilution 1:100; catalog no., M362629-2; Dako, Glostrup, Denmark) and anti-p16 (clone JC8; dilution 1:100; catalog no., sc-56330; Santa Cruz Biotechnology, Inc., Dallas, TX, USA) antibodies was performed on $1-\mu \mathrm{m}$ sections prepared from paraffin blocks using a fully automated slide preparation system (BenchMark XT System; Ventana Medical Systems Inc., Tucson, AZ, USA). The slides were evaluated by two independent pathologists. IMP3 staining was evaluated semi-quantitatively using a system for staining intensity, as described previously (24): 0 (no staining); 1+ (moderate to strong staining in $<25 \%$ of cells or weak staining to any extent); $2+$ (moderate to strong staining in $25-50 \%$ of cells); and $3+$ (moderate to strong staining in $>50 \%$ of cells).

Tumors were then grouped into two groups of IMP3-(negative/weak staining, 0/1+) and IMP3+ (moderate/strong staining, $2+/ 3+$ ). Tumors were considered
Table I. Association between IMP3 and p16 expression in 156 head and neck squamous cell carcinomas $(\mathrm{P}=0.61)$.

\begin{tabular}{lllr}
\hline & \multicolumn{2}{c}{ IMP3 } \\
\cline { 2 - 3 } p16 & Negative & Positive & Total \\
\hline Negative & $51(50)$ & $51(50)$ & $102(100)$ \\
Positive & $24(44.4)$ & $30(55.6)$ & $54(100)$ \\
Total & $75(48.1)$ & $81(51.9)$ & $156(100)$ \\
\hline
\end{tabular}

Data are presented as n (\%). IMP3, insulin-like growth factor II mRNA binding protein 3 .

positive for p16 when strong nuclear and cytoplasmic staining was present in $>50 \%$ of cells (5). The validity of the anti-p16 antibody as a surrogate marker for HPV was confirmed by comparing it to the CINtech protocol involving a large cohort of routine specimens stained in parallel with both antibodies (the anti-p16 antibody, clone JC8, and the CINtech protocol) at the Institute of Pathology, University Hospital, Friedrich-Alexander University Erlangen-Nuremberg (Erlangen, Germany) (Agaimy et al, unpublished data).

$H P V$ status. To determine whether there is an association between the expression of the two biomarkers and HPV status, 38 tumors were randomly selected to represent the four possible patterns of p16 and IMP3 expression: p16+/IMP3-(n=9), p16-/IMP3+ $(\mathrm{n}=10), \mathrm{p} 16+/ \mathrm{IMP} 3+(\mathrm{n}=11)$ and p16-/IMP3- $(\mathrm{n}=8)$. Molecular testing for HPV in the 38 cases was performed as described previously (34).

Statistical analysis. Fisher's exact test was used to test for associations in contingency tables (or alternatively the $\chi^{2}$ test was used for tables with at least three rows and three columns). The sensitivity, specificity and predictive values of potential markers were determined based on $2 \times 2$-tables and are presented together with their exact $95 \%$ confidence intervals (CIs). The statistical analyses were performed with SAS 9.3 (SAS Institute INC., Cary, NC, USA).

\section{Results}

IMP3 expression in HNSCC. Of the 156 HNSCCs, 81 (51.9\%) were positive for IMP3 (Table I). There was an association between IMP3 positivity and HNSCC localization $(\mathrm{P}=0.022)$, with $50(61.7 \%)$ of the OPSCCs being positive for IMP3 compared with only $16(36.4 \%)$ of the oral cavity SCC cases (Table II). No positive association between IMP3 expression and HPV status was observed; however, a trend towards a negative association was apparent $(\mathrm{P}=0.053$; Table III). Expression of IMP3 was strictly cytoplasmic, as compared with the characteristic combined nucleocytoplasmic expression of p16 (Fig. 1).

To address the question of whether IMP3 is a suitable surrogate marker for HPV infection, we calculated the sensitivity (i.e. the proportion of HPV positives that were correctly identified) specificity (i.e. the proportion of HPV 
Table II. Immunoprofiles of IMP3 and p16 in association with localization of oral squamous cell carcinomas.

\begin{tabular}{|c|c|c|c|c|c|c|}
\hline Marker & Oral cavity & Oro-pharynx & Naso-pharynx & Hypo-pharynx & Larynx & P-value \\
\hline IMP3 & & & & & & 0.022 \\
\hline Negative & $28(63.6)$ & $31(38.3)$ & $1(16.7)$ & $6(60.0)$ & $9(60.0)$ & \\
\hline Positive & $16(36.4)$ & $50(61.7)$ & $5(83.3)$ & $4(40.0)$ & $6(40.0)$ & \\
\hline p16 & & & & & & $<0.001$ \\
\hline Negative & $39(88.6)$ & $38(46.9)$ & $5(83.3)$ & $7(70.0)$ & $13(86.7)$ & \\
\hline Positive & $5(11.4)$ & $43(53.1)$ & $1(16.7)$ & $3(30.0)$ & $2(13.3)$ & \\
\hline
\end{tabular}

Data are presented as n (\%). Percentages in columns add up to $100 \%$ within a marker. IMP3, insulin-like growth factor II mRNA binding protein 3.

Table III. Sensitivities, specificities, negative predictive values and positive predictive values of IMP3 and/or p16 expression for predicting the human papillomavirus status in head and neck squamous cell carcinoma.

\begin{tabular}{lccccc}
\hline Marker combination & P-value & $\begin{array}{c}\text { Sensitivity } \\
(95 \% \mathrm{CI})\end{array}$ & $\begin{array}{c}\text { Specificity } \\
(95 \% \mathrm{CI})\end{array}$ & $\begin{array}{c}\text { Negative predictive value } \\
(95 \% \mathrm{CI})\end{array}$ & $\begin{array}{c}\text { Positive predictive value } \\
(95 \% \mathrm{CI})\end{array}$ \\
\hline IMP3 & 0.053 & $0.47(0.28-0.66)$ & $0.13(0.01-0.53)$ & $0.06(0.01-0.29)$ & $0.67(0.43-0.85)$ \\
p16 & 0.017 & $0.63(0.44-0.80)$ & $0.88(0.47-0.99)$ & $0.39(0.17-0.64)$ & $0.95(0.75-0.99)$ \\
Combination IMP3/p16 & 0.391 & $0.13(0.01-0.53)$ & $0.77(0.58-0.90)$ & $0.13(0.01-0.53)$ & $0.77(0.58-0.90)$ \\
\hline
\end{tabular}

CI, confidence interval; IMP3, insulin-like growth factor II mRNA binding protein 3.

Table IV. Immunoprofiles of the combination of IMP3 and p16 in association with the localization of oral squamous cell carcinomas $(\mathrm{P}<0.001)$.

\begin{tabular}{|c|c|c|c|c|c|c|}
\hline Marker combination & Oral cavity & Oro-pharynx & Naso-pharynx & Hypo-pharynx & Larynx & Total \\
\hline IMP3 \& p16 negative & $25(56.8)$ & $12(14.8)$ & $1(16.7)$ & $5(50.0)$ & $8(53.3)$ & $51(32.7)$ \\
\hline Either IMP3 positive or p16 positive & $17(38.6)$ & $45(55.6)$ & $4(66.7)$ & $3(30.0)$ & $6(40.0)$ & $75(48.1)$ \\
\hline IMP3 \& p16 positive & $2(4.6)$ & $24(29.6)$ & $1(16.6)$ & $2(20.0)$ & $1(16.7)$ & $30(19.2)$ \\
\hline Total & $44(100)$ & $81(100)$ & $6(100)$ & $10(100)$ & $15(100)$ & $156(100)$ \\
\hline
\end{tabular}

Data are presented as n (\%). Percentages add up to $100 \%$ in each column. IMP3, insulin-like growth factor II mRNA binding protein 3.

negatives that were correctly identified) negative predictive value (NPV; i.e. the proportion of IMP3 negatives that were also HPV negative) and positive predictive value (PPV; i.e. the proportion of IMP3 positives that were also HPV positive) were calculated. For IMP3, the sensitivity was $0.47(95 \%$ $\mathrm{CI}=0.28-0.66)$, the specificity was 0.13 (95\% $\mathrm{CI}=0.01-0.53)$, the NPV was $0.06(95 \% \mathrm{CI}=0.01-0.29)$ and the PPV was 0.67 (95\% CI=0.43-0.85) (Table III).

p16 expression in HNSCC. Of the 156 HNSCCs, 54 (34.6\%) were positive for p16 (Table I). The expression of p16 was observed most frequently in OPSCCs (53.1 vs. $\leq 30 \%$ for other localization; $\mathrm{P}<0.001$; Table II). p16 expression was also significantly associated with HPV status $(\mathrm{P}=0.017)$. The specificity of p16 for HPV status in the present study was 0.88 (95\% CI=0.47-0.99), combined with a sensitivity of 0.63 (95\% CI=0.44-0.80), an NPV of 0.39 (95\% CI=0.17-0.64) and a PPV of 0.95 (95\% CI=0.75-0.99) (Table III). Although a cutoff of $>50 \%$ for positive p16 results was applied, as in a previous study (5), all positive cases showed expression of $80-100 \%$ in tumor cells, which is in line with the known pattern of p16 in OPSCC (5).

Comparative expression of IMP3 and p16 in OPSCC vs. non-OPSCC and correlation with HPV status. IMP3 and p16 were expressed in $81(51.9 \%)$ and $54(34.6 \%)$ of the HNSCC cases, respectively (Table I). When combining the results of IMP3 and p16, it was found that $75(48.1 \%)$ were positive for either one of the markers, 51 (32.7\%) were negative for both markers and 30 (19.2\%) were positive for both markers. These findings were associated with the localization of the tumors $(\mathrm{P}<0.001$; Table IV). Notably, 25/44 $(56.8 \%)$ of the oral cavity SCCs were negative for IMP3 and p16, as compared with only 12/81 (14.81\%) of the OPSCCs (Table IV). 
HE p16 IMP3
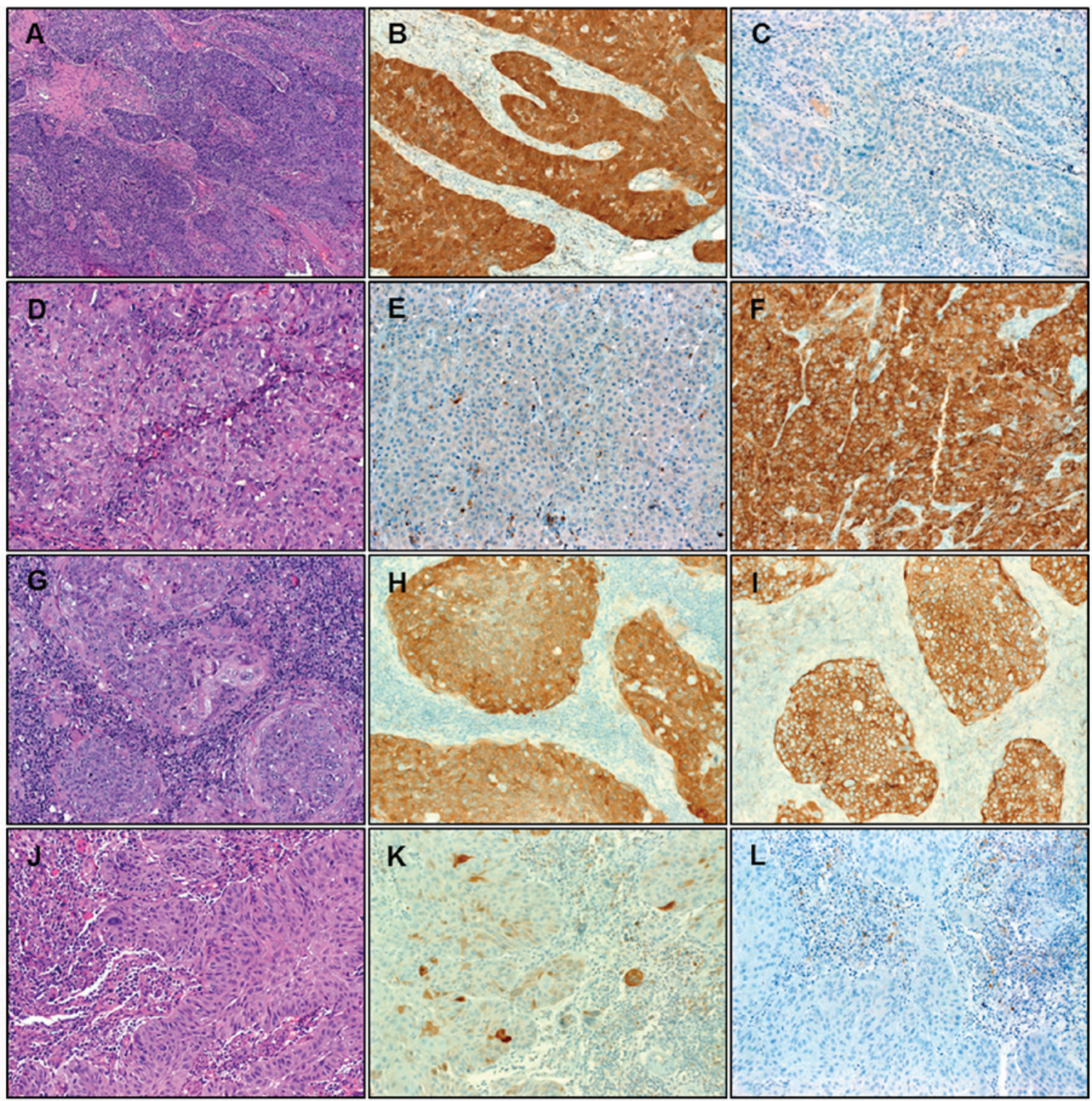

Figure 1. Immunohistochemical analysis of HNSCC. Representative examples of the four different expression patterns of p16 and IMP3 in HNSCC. (A-C) HNSCC showing strong expression of p16 but not IMP3. (D-F) HNSCC negative with p16 and strongly positive for IMP3. (G-I) HNSCC expressing both markers. (J-L) HNSCC negative for both markers. HNSCC, head and neck squamous cell carcinoma; IMP3, insulin-like growth factor II mRNA binding protein 3; HE, hematoxylin and eosin. Magnification, x200.

Although the combination of the expression of IMP3 and p16 was not significantly associated with the HPV status $(\mathrm{P}=0.391)$, it is interesting to note that $10 / 11(90.9 \%)$ of the tumors coexpressing IMP 3 and p16 were HPV positive by polymerase chain reaction (Table III).

In the present study, the specificity for the combination of p16 and IMP3 in predicting the HPV status was 0.77 (95\% $\mathrm{CI}=0.58-0.90)$, combined with a sensitivity of $0.13(95 \%$ $\mathrm{CI}=0.01-0.53)$, an NPV of 0.13 (95\% $\mathrm{CI}=0.01-0.53)$ and a PPV of 0.77 (95\% CI=0.58-0.90). These results suggest that the variable combinations of p16 and/or IMP3 expression do not qualify as predictive markers for HPV infection in HNSCC (Table III).

HPV status. In the subgroup analyzed for HPV status ( $\mathrm{n}=38$ cases), 30 (79.0\%) were HPV positive, which showed an association with the localization ( $\mathrm{P}=0.029)$; all $19(100 \%)$ OPSCCs were HPV positive (data not shown).

\section{Discussion}

Since its discovery in 1997, several studies have investigated the expression status of IMP3 in various neoplasms and in normal tissues in order to understand its role in diseases (9). IMP3 is overexpressed in human cancer, but it also has a ubiquitous role in early embryogenesis, including in the development of the intestine, thymus, pancreas and kidneys (8). Functional studies have shown that IMP3 possibly influences tumor cell adhesion and enhances invasive growth in cancer $(11,12)$. However, currently, the actual role of IMP3 in the modulation of tumor cell function and invasiveness remains poorly understood. 
Although extensively studied in several cancer types, only a few studies have previously investigated the expression status of IMP3 in HNSCC. IMP3 overexpression in SCC of the oral cavity was shown to be an independent predictive marker for an advanced clinical stage, lymph node metastasis and as a prognostic indicator (29-31). Furthermore, it has been reported to be an indicator of a poor prognosis and a marker of enhanced malignancy in SCC of the tongue and larynx (32-35). In addition, it has been shown to be expressed in certain malignant salivary gland tumors (36).

Little is known about the interactions between IMP3 and p16 expression in cancer. A few studies investigating carcinomas of the uterine cervix focused on this subject. Li et al (16) reported that a combination of these markers may be helpful in the distinction of adenocarcinoma from benign endocervical glands in situ. Furthermore, another study found that the combination of p16/IMP3 expression improved the discrepant results between cytological and histological diagnoses (37). In HIV-positive patients, IMP3 showed a higher sensitivity than p16 for identifying patients at risk of progression and recurrence of cervical intraepithelial neoplasia (38)

To the best of our knowledge, no previous study has investigated the expression of IMP3 in comparison with p16 and/or HPV status in HNSCC, which was the aim of the present pilot study. The present study analyzed and compared the expression status of IMP3 and p16 in a cohort of HNSCCs enriched with HPV-positive OPSCCs. The current study showed that IMP3 and p16 were frequently expressed in SCCs of the head and neck; in particular, more frequently in oropharyngeal carcinomas, as compared with other sites.

The present study demonstrated that IMP3 was more frequently expressed in HNSCC than p16 (51.9 vs. 34.6\%, respectively). Furthermore, IMP3 expression was significantly associated with localization to the oropharynx $(\mathrm{P}=0.022)$, as it was expressed in $61.7 \%$ of OPSCC cases compared with only $41.3 \%$ of SCCs from other head and neck sites.

In contrast to the results observed for p16, a trend towards a negative association between IMP3 expression and HPV status was observed in the present study. Therefore, the potential role of IMP3 as a marker for aggressiveness in HPV-related and non-HPV-related HNSCC, as well as its prognostic impact, require verification in larger cohorts with an extended follow-up period.

A previous study showed promising results in support of the use of IMP3 as a potential vaccination therapy in patients with HNSCC (39). A new Phase II study using IMP3 as a vaccination therapy in patients with HNSCC showed an immune response and an improved overall survival (39). These developments underline the relevance of further in-depth studies in the future to determine the exact roles of IMP3 in different molecular and etiological subtypes of HNSCC.

In summary, the present study analyzed and compared the expression status of p16 and IMP3, as well as the HPV status, in a cohort of HNSCCs enriched with HPV-positive OPSCC. The results of the present study highlighted a potential role for IMP3 as a biomarker in HPV-positive HNSCC. Further studies involving larger cohorts with an extended follow-up are required to validate the value of combined p16/IMP3 expression as predictive prognostic and/or therapeutic biomarkers in HNSCC.

\section{References}

1. Barnes L, Eveson JW, Reichart P and Sidransky D (eds): World Health Organization Classification of Tumours. Pathology and Genetics of Head and Neck Tumours. Lyon, France: IARC Press, 46-50, 2005.

2. Jo VY, Mills SE, Stoler MH and Stelow EB: Papillary squamous cell carcinoma of the head and neck: Frequent association with human papillomavirus infection and invasive carcinoma. Am J Surg Pathol 33: 1720-1724, 2009.

3. Benevolo M, Mottolese M, Marandino F, Vocaturo G, Sindico R, Piperno G, Mariani L, Sperduti I, Canalini P, Donnorso RP and Vocaturo A: Immunohistochemical expression of p16(INK4a) is predictive of HR-HPV infection in cervical low-grade lesions. Mod Pathol 19: 384-391, 2006.

4. Lewis JS Jr, Thorstad WL, Chernock RD, Haughey BH, Yip JH, Zhang Q and El-Mofty SK: p16 positive oropharyngeal squamous cell carcinoma: An entity with a favorable prognosis regardless of tumor HPV status. Am J Surg Pathol 34: 1088-1096, 2010.

5. Mehrad M, Carpenter DH, Chernock RD, Wang H, Ma XJ, Luo Y, Luo J, Lewis JS Jr and El-Mofty SK: Papillary squamous cell carcinoma of the head and neck: Clinicopathologic and molecular features with special reference to human papillomavirus. Am J Surg Pathol 37: 1349-1356, 2013.

6. Cai C, Chernock RD, Pittman ME, El-Mofty SK, Thorstad WL and Lewis JS Jr: Keratinizing-type squamous cell carcinoma of the oropharynx: p16 overexpression is associated with positive high-risk HPV status and improved survival. Am J Surg Pathol 38: 809-815, 2014.

7. Müeller-Pillasch F, Lacher U, Wallrapp C, Micha A, Zimmerhackl F, Hameister H, Varga G, Friess H, Büchler M, Beger HG, et al: Cloning of a gene highly overexpressed in cancer coding for a novel $\mathrm{KH}$-domain containing protein. Oncogene 14: 2729-2733, 1997.

8. Mueller-Pillasch F, Pohl B, Wilda M, Lacher U, Beil M, Wallrapp $\mathrm{C}$, Hameister $\mathrm{H}$, Knöchel $\mathrm{W}$, Adler $\mathrm{G}$ and Gress TM: Expression of the highly conserved RNA binding protein KOC in embryogenesis. Mech Dev 88: 95-99, 1999.

9. Nielsen FC, Nielsen J and Christiansen J: A family of IGF-II mRNA binding proteins (IMP) involved in RNA trafficking. Scand J Clin Lab Invest Suppl 234: 93-99, 2001.

10. Monk D, Bentley L, Beechey C, Hitchins M, Peters J, Preece MA, Stanier P and Moore GE: Characterisation of the growth regulating gene IMP3, a candidate for Silver-Russell syndrome. J Med Genet 39: 575-581, 2002.

11. Liao B, Hu Y, Herrick DJ and Brewer G: The RNA-binding protein IMP-3 is a translational activator of insulin-like growth factor II leader-3 mRNA during proliferation of human K562 leukemia cells. J Biol Chem 280: 18517-18524, 2005.

12. Vikesaa J, Hansen TV, Jønson L, Borup R, Wewer UM, Christiansen $\mathrm{J}$ and Nielsen FC: RNA-binding IMPs promote cell adhesion and invadopodia formation. EMBO J 25: 1456-1468, 2006.

13. Hoffmann NE, Sheinin Y, Lohse CM, Parker AS, Leibovich BC, Jiang $\mathrm{Z}$ and Kwon ED: External validation of IMP3 expression as an independent prognostic marker for metastatic progression and death for patients with clear cell renal cell carcinoma. Cancer 112: 1471-1479, 2008.

14. Jiang Z, Lohse CM, Chu PG, Wu CL, Woda BA, Rock KL and Kwon ED: Oncofetal protein IMP3: A novel molecular marker that predicts metastasis of papillary and chromophobe renal cell carcinomas. Cancer 112: 2676-2682, 2008.

15. Li C, Rock KL, Woda BA, Jiang Z, Fraire AE and Dresser K: IMP3 is a novel biomarker for adenocarcinoma in situ of the uterine cervix: An immunohistochemical study in comparison with p16(INK4a) expression. Mod Pathol 20: 242-247, 2007.

16. Li C, Zota V, Woda BA, Rock KL, Fraire AE, Jiang Z, Lu D, $\mathrm{Xu}$ B, Dresser K, Lutman CV and Fischer AH: Expression of a novel oncofetal mRNA-binding protein IMP3 in endometrial carcinomas: Diagnostic significance and clinicopathologic correlations. Mod Pathol 20: 1263-1268, 2007.

17. Lu D, Vohra P, Chu PG, Woda B, Rock KL and Jiang Z: An oncofetal protein IMP3: A new molecular marker for the detection of esophageal adenocarcinoma and high-grade dysplasia. Am J Surg Pathol 33: 521-525, 2009.

18. Pryor JG, Bourne PA, Yang Q, Spaulding BO, Scott GA and $\mathrm{Xu} \mathrm{H}$ : IMP-3 is a novel progression marker in malignant melanoma. Mod Pathol 21: 431-437, 2008. 
19. Pryor JG, Simon RA, Bourne PA, Spaulding BO, Scott GA and $\mathrm{Xu} \mathrm{H}$ : Merkel cell carcinoma expresses $\mathrm{K}$ homology domain-containing protein overexpressed in cancer similar to other high-grade neuroendocrine carcinomas. Hum Pathol 40: 238-243, 2009

20. Sitnikova L, Mendese G, Liu Q, Woda BA, Lu D, Dresser K, Mohanty S, Rock KL and Jiang Z: IMP3 predicts aggressive superficial urothelial carcinoma of the bladder. Clin Cancer Res 14: 1701-1706, 2008.

21. Xu H, Bourne PA, Spaulding BO and Wang HL: High-grade neuroendocrine carcinomas of the lung express $\mathrm{K}$ homology domain containing protein overexpressed in cancer but carcinoid tumors do not. Hum Pathol 38: 555-563, 2007.

22. Strehl JD, Hoegel J, Hornicek I, Hartmann A and Riener MO: Immunohistochemical expression of IMP3 and p53 in inflammatory lesions and neoplastic lesions of the gastric mucosa. Int J Clin Exp Pathol 7: 2091-2101, 2014.

23. Wachter DL, Kristiansen G, Soll C, Hellerbrand C, Breuhahn K, Fritzsche F, Agaimy A, Hartmann A and Riener MO: Insulin-like growth factor II mRNA-binding protein 3 (IMP3) expression in hepatocellular carcinoma. A clinicopathological analysis with emphasis on diagnostic value. Histopathology 60: 278-286, 2012.

24. Wachter DL, Schlabrakowski A, Hoegel J, Kristiansen G, Hartmann A and Riener MO: Diagnostic value of immunohistochemical IMP3 expression in core needle biopsies of pancreatic ductal adenocarcinoma. Am J Surg Pathol 35: 873-877, 2011

25. Yantiss RK, Woda BA, Fanger GR, Kalos M, Whalen GF Tada H, Andersen DK, Rock KL and Dresser K: KOC ( $\mathrm{K}$ homology domain containing protein overexpressed in cancer): A novel molecular marker that distinguishes between benign and malignant lesions of the pancreas. Am J Surg Pathol 29: 188-195, 2005.

26. Zhao H, Mandich D, Cartun RW and Ligato S: Expression of $\mathrm{K}$ homology domain containing protein overexpressed in cancer in pancreatic FNA for diagnosing adenocarcinoma of pancreas. Diagn Cytopathol 35: 700-704, 2007.

27. Riener MO, Fritzsche FR, Clavien PA, Pestalozzi BC, Probst-Hensch N, Jochum W and Kristiansen G: IMP3 expression in lesions of the biliary tract: A marker for high-grade dysplasia and an independent prognostic factor in bile duct carcinomas. Hum Pathol 40: 1377-1383, 2009.

28. Walter O, Prasad M, Lu S, Quinlan RM, Edmiston KL and Khan A: IMP3 is a novel biomarker for triple negative invasive mammary carcinoma associated with a more aggressive phenotype. Hum Pathol 40: 1528-1533, 2009.

29. Lin CY, Chen ST, Jeng YM, Yeh CC, Chou HY, Deng YT, Chang CC and Kuo MY: Insulin-like growth factor II mRNA-binding protein 3 expression promotes tumor formation and invasion and predicts poor prognosis in oral squamous cell carcinoma. J Oral Pathol Med 40: 699-705, 2011.
30. Li S, Cha J, Kim J, Kim KY, Kim HJ, Nam W and Cha IH: Insulin-like growth factor II mRNA-binding protein 3: A novel prognostic biomarker for oral squamous cell carcinoma. Head Neck 33: 368-374, 2011

31. Kim KY, Li S, Cha JD, Zhang X and Cha IH: Significance of molecular markers in survival prediction of oral squamous cell carcinoma. Head Neck 34: 929-936, 2012.

32. Li HG, Han JJ, Huang ZQ, Wang L, Chen WL and Shen XM: IMP3 is a novel biomarker to predict metastasis and prognosis of tongue squamous cell carcinoma. J Craniofac Surg 22: 2022-2025, 2011

33. Clauditz TS, Wang CJ, Gontarewicz A, Blessmann M, Tennstedt P, Borgmann K, Tribius S, Sauter G, Dalchow C, Knecht R, et al: Expression of insulin-like growth factor II mRNA-binding protein 3 in squamous cell carcinomas of the head and neck. J Oral Pathol Med 42: 125-132, 2013.

34. Chen K, Cornejo KM, Ye W, Wu Q, Liang J and Jiang Z: Oncofetal protein IMP3: A new diagnostic biomarker for laryngeal carcinoma. Hum Pathol 44: 2126-2131, 2013.

35. Knör M, Tziridis K, Agaimy A, Zenk J and Wendler O: Human Papillomavirus (HPV) Prevalence in Nasal and Antrochoanal Polyps and association with clinical data. PLoS One 10: $\mathrm{e} 0141722,2015$.

36. Ismerim AB, Ferreira SV, Lessa AM, Pereira Júnior AS, Gurgel CA, Coutinho-Camillo CM, Soares FA, Vilas-Bôas DS, Vidal MT and Santos JN: Insulin-like growth factor II messenger RNA-binding protein 3 in Salivary Gland tumors. Appl Immunohistochem Mol Morphol 24: 422-426, 2016.

37. Wei Q, Fu B, Liu J, Xu J and Zhao T: Combined detection of p16(INK4a) and IMP3 increase the concordance rate between cervical cytologic and histologic diagnosis. Int J Clin Exp Pathol 6: 1549-1557, 2013.

38. Del Gobbo A, Bonoldi E, Cribiù FM, Franceschetti I, Matinato C, Fiori S, Gianelli U and Bosari S: Insulin-like growth factor II mRNA binding protein 3 (IMP3) expression in cervical intraepithelial neoplasia and its relationship with HIV-infection status. Sex Health 12: 22-26, 2015.

39. Yoshitake Y, Fukuma D, Yuno A, Hirayama M, Nakayama $H$, Tanaka T, Nagata M, Takamune Y, Kawahara K, Nakagawa Y, et al: Phase II clinical trial of multiple peptide vaccination for advanced head and neck cancer patients revealed induction of immune responses and improved OS. Clin Cancer Res 21: 312-321, 2015 . 